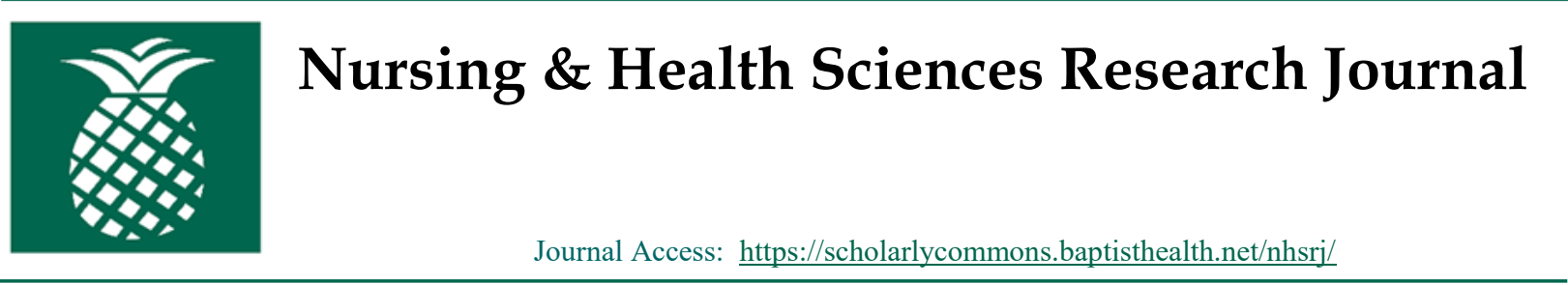

\title{
Navigating a Difficult Transition in Pulmonary Arterial Hypertension: A Clinical Case Study
}

Tina Hyman, MSN, RN-BC; Michael Salinero, BSN, RN; Javier Jimenez, MD, PhD, FACC;

Margarita Pallares, MSN, APRN, AGACNP-BC; Maribel Matos , MSN, APRN, AANP-BC

\section{ABSTRACT}

Over the past 20 years, Pulmonary Arterial Hypertension (PAH) patients and providers have seen an increase in options to treat PAH. Currently, there are choices for the delivery of medications approved to treat patients with PAH where at one point the only option was intravenous (IV) therapy. This case study details the transition from IV Epoprostenol to oral Treprostinil for the treatment of a 53 year old female diagnosed with severe Pulmonary Arterial Hypertension whose quality of life became unbearable by the IV medication delivery route. This patient was a synchronized swimmer in her youth and her passion for water activities continued throughout her short life. After her diagnosis of severe PAH, her life abruptly changed as she was placed on triple therapy, which consisted of an endothelin receptor antagonist (ERA), a phosphodiesterase-5 inhibitor (pde-5), and the IV epoprostenol. The patient experienced severe side effects that negatively impacted her ability to resume her activities of daily life and swimming. Although she remained hemodynamically stable on IV therapy, her poor quality of life and severe depression convinced her cardiologist that transitioning to an oral treprostinil would be the only option for her survival. At the time, there was a lack of clinical trials or guidelines to support the transition from IV to oral prostacyclins to follow, so an interdisciplinary team approach needed to be taken to ensure that the safety and efficacy of the transition would be maintained.

Keywords: Pulmonary arterial hypertension (PAH), Transition, Epoprostenol, Treprostinil, Quality of life

\section{BACKGROUND}

The World Health Organization has categorized Pulmonary Hypertension into five groups: Pulmonary Arterial Hypertension, Pulmonary Hypertension due to left heart disease, Pulmonary Hypertension due to lung disease, Pulmonary Hypertension due to chronic blood clots in the lungs, and Pulmonary Hypertension due to unknown causes (Pulmonary Hypertension Association, 2019). PAH is diagnosed during a right heart catheterization and is characterized by the remodeling, narrowing and elimination of pulmonary arteries caused by endothelial cell proliferation, inflammation of the vascular wall and fibrosis which elevates the pulmonary pressures (Hambly, 2016). It is a rare but serious condition affecting as few as 5 in 1 million adults with a 5 year survival rate of just $37 \%$ as recorded in the 1980's NIH registry (McLaughlin, 2013).
The associated symptoms are disabling to the patient and include dyspnea, fatigue, syncope, and chest pain leading to premature death due to their risk for developing right ventricular failure (Hambly, 2016 ).

It was Aminorex (Fen/phen), an appetite suppressant, which caused an epidemic of the disease in 1965 which led to the withdrawal of Fen/Phen from the market in 1997 (Anderson, 2016). It wasn't until the 1990's that a treatment care plan including warfarin in combination with high dose calcium channel blockers started being prescribed for the treatment of $\mathrm{PH}$. Then, a clinical trial demonstrated that the use of long-term intravenous (IV) prostacyclin improved the hemodynamics and functional class in this patient population (McLaughlin, 2013). Following the study of IV prostacyclins, inhaled and oral prostacyclins were investigated; in 2013, the Food and Drug Administration (FDA) approved the first oral prostacyclin for the treat- 
ment of PAH (Anderson, 2016). The oral drug was in development for 17 years and offered another route of administration for patients requiring prostacyclin therapy (United Therapeutics, 2013). Currently, there are 14 approved medications for the treatment of PH. At one point the treatment option for $\mathrm{PH}$ was exclusively IV therapy but now the delivery options have expanded to include both oral and inhalation administration routes.

More oral and inhaled prostacyclins or prostacyclin analogs have become available in recent years. Recently successful transitions from IV prostacyclin to one of the oral/inhaled equivalents have been reported, but at the time of this report, there were no published guidelines to transition patients from IV epoprostenol to oral treprostinil to refer to. Although both drugs are similar, epoprostenol differs from treprostinil with its shorter half -life, no greater than six minutes, and the absence of an oral equivalent. Both drugs, independently, have been shown to be effective in the treatment of pulmonary hypertension (Saito, 2015).

The use of central lines to deliver prostacyclin therapy comes with many expected side-effects as well as complications. Flushing, sinus congestion, GI upset, diarrhea, generalized edema, insertion site discomfort, generalized pain, and headaches are the most common side effects which effects patient's quality of life (Hanlon, 2017). It is reasonable that patients, like the patient in this case study, who suffer with severe side effects, experience a decrease in quality of life. The treatment regimen for each patient is highly individualized according to the patient's tolerance of each titration increase. The following case study details the seven week transition schedule that was used to wean the patient off of IV onto an oral treprostinil and challenges the healthcare team had to overcome to help this patient achieve greater independence and better quality of life.

\section{CASE PRESENTATION}

A 53-year-old female diagnosed with severe Pulmonary Arterial Hypertension by right heart catheterization in 2012. It is believed that this patient developed PAH by using the appetite suppressant Fen-Phen. In 2012 the patient was placed on triple therapy which included a phosphodiesterase inhibitor (PDE-5), an endothelin receptor antagonist (ERA), and IV epoprostenol. The patient was referred to a $\mathrm{PH}$ specialist for further evaluation and treatment. The patient experienced severe side-effects from the IV infusion in- cluding: pronounced facial flushing, sinus congestion, GI upset, diarrhea, generalized edema, and headache. Other complaints included central catheter pain, shortness of breath on exertion, depression, and continuous oxygen dependence. The patient remained medically stable on the IV therapy but due to the risk for line infection, safety, and diminished quality of life, the physician and patient decided to transition from the IV epoprostenol to oral treprostinil.

Establishing a transition protocol from IV epoprostenol to oral treprostinil in the absence of clear guidelines and in an outpatient setting was challenging. A health care team consisting of a pharmacist, nurses, cardiologist, pulmonologist, and drug manufacturer $\mathrm{RN}$ representative were all consulted and contributed to the patient's titration schedule. Each specialty discussed treatment options and potential complications. Once the choice of oral medication was identified, a clinical pharmacist assembled a transition schedule using contributions given by the planning team and limited data which was available on the topic. The weekly titrations were performed under the close observation of the cardiologist whether in the medical office, at home with the drug $\mathrm{RN}$ representative, or in the outpatient clinic which specializes in PH. The patient's physical and mental status were discussed, evaluated, and well documented during the seven week period. A slow transition schedule was used to accommodate the patient and secure her safety. The patient was seen at the cardiologist office as well as the heart failure outpatient clinic alternating weekly and had phone access to all members of the team 24/7. The dosing schedule using dual therapy during the seven day titration period is shown in Table 1 . The patient was getting $20 \mathrm{ng} / \mathrm{kg} / \mathrm{min}$ of the IV epoprostenol prior to the titration.

\section{DISCUSSION}

This case represents a difficult but successful transition from intravenous epoprostenol to oral treprostinil despite the lack of guidelines. The patient was able to transition to oral treprostinil $14 \mathrm{mg} / \mathrm{p}$.o./daily and stayed medically stable. Furthermore, her exercise tolerance increased and she was able to resume activities, such as swimming and other hobbies that were prohibited once IV therapy had been initiated improving her quality of life and renewing her sense of hope. Since this case study, there has been many successful week-long transitions published with improved exercise tolerance and patient outcomes (Gleason, 2015). 
Table 1

Epoprostenol IV to Treprostinil Oral Transition Schedule

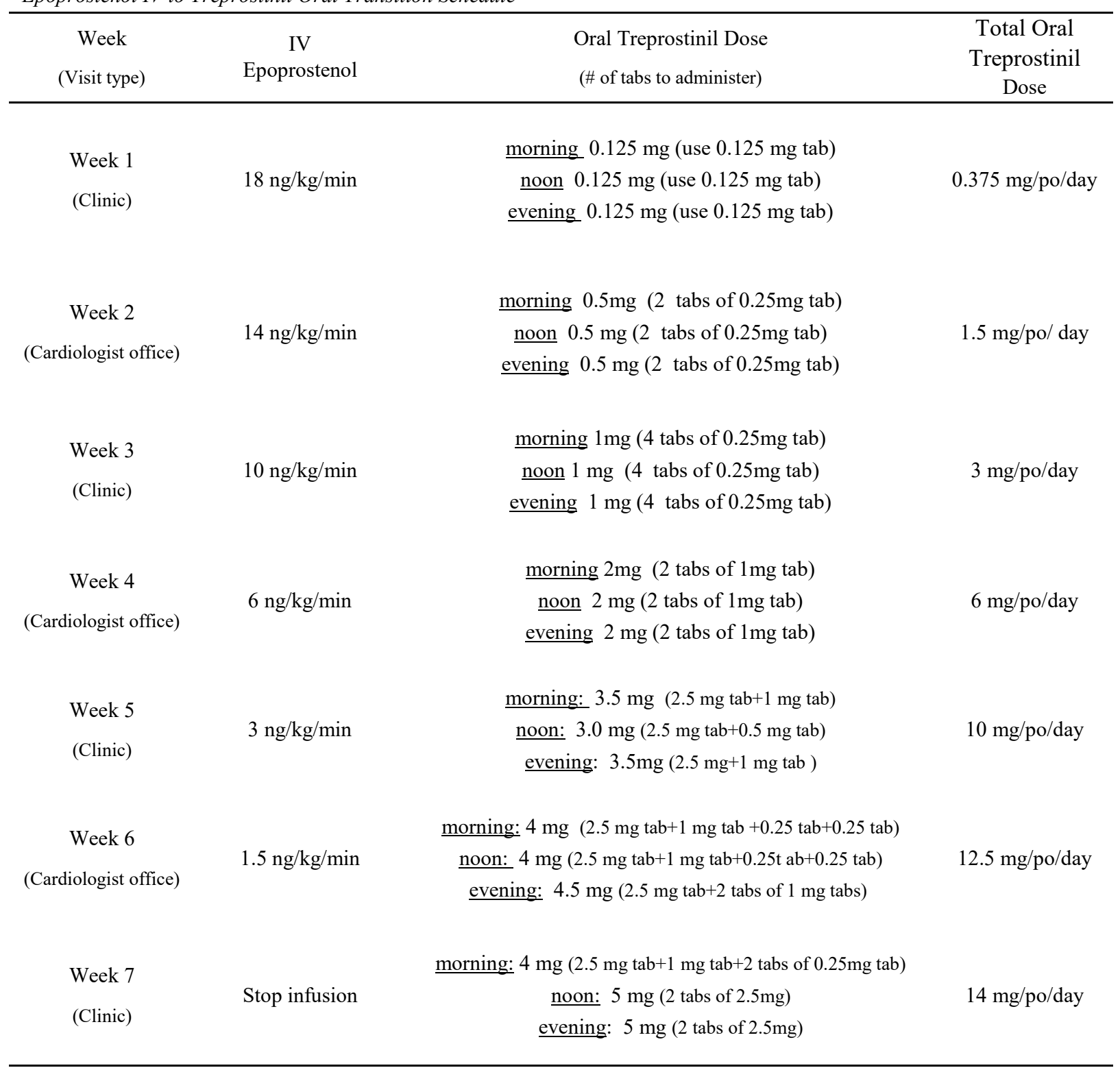

Similarly, there have been successful, safe, and cost efficient transitions from IV epoprostenol to oral $\mathrm{PH}$ prostacyclin therapies in the outpatient setting (Morris KL, 2018). However, at the time of this case, the healthcare team had to work very careful and diligently regardless of the lack of treatment guidelines, to make a quick decision to provide this patient with a meaningful and improved quality of life.

Currently, there are published criteria that identify ideal candidates for IV to oral transitions. These patients have to show hemodynamic, clinical, and physiological signs of stability in order to achieve a successful transition (Coons, 2016; Sofer, 2017). This case report does not provide data on the long-term success from an IV epoprostenol to oral treprostinil transition, however, this patient was able to transition and maintain her physiological status as well as report a better quality of life. 


\section{CONCLUSION}

This case study demonstrates that making sound treatment decisions can be difficult at times but the goal of the treatment can be accomplished with strong collaboration, compassion, and respect for the individual's quality of life. The transition incorporated the expertise of the multidisciplinary team including the pharmacist, nurses, cardiologist, pulmonologist, and drug manufacturer's RN representative in order to establish a safe plan of care that addressed the individual needs of this patient. The best decision-making for medical treatments is made when healthcare providers take into account patients' quality of life.

\section{DECLARATION OF INTEREST}

The authors report no conflicts of interest. The authors alone are responsible for the content and writing of the paper.

\section{AUTHORS}

\section{Tina Hyman, MSN, RN-BC}

Clinical Nurse Educator 1

South Miami Hospital Heart Rhythm Center

6200 SW 73 St. South Miami, Fl. 33143

Correspondence regarding

this paper can be directed at TinaH@,baptisthealth.net

\section{Michael Salinero, BSN, RN}

Nurse Manager- Heart Rhythm Center

South Miami Hospital Heart Rhythm Center

6200 SW 73 St. South Miami, Fl. 33143

Francisco Javier Jimenez-Carcamo MD, PhD, FACC.

Director of Advanced Heart Failure and Pulmonary

Hypertension

6200 Sunset Dr. Ste. 401

South Miami, F1. 33143

Margarita Pallares, MSN, APRN, AGACNP-BC

Adult Gerontology Acute Care Nurse Practitioner

South Miami Hospital Heart Rhythm Center

6200 SW 73 St. South Miami, Fl. 33143

Maribel Matos, MSN, APRN, AANP-BC

Nurse Practitioner

South Miami Hospital Heart Rhythm Center

6200 SW 73 St. South Miami, FL. 33143

\section{REFERENCES}

Anderson, R. M. (2016). Pulmonary hypertension: evolution of pulmonary arterial hypertension and chronic thromboembolic pulmonary hy pertension. Journal of Thoracic Disease, S562 -S565.

Badesch, D. (1997). Clinical trials in pulmonry hypertension. Annual review of medicine (00664219), 48, 399.

Coons, C. J. (2016). Oral treprostinil for the treatment of arterial hypertension in patient transitioned from parenteral or inhaled prostacyclins: Case series and treatment protocol. Pulmonary Circulation, (6), 10.1086/685111.

Gleason, J. D. (2015). The Rapid Initiation, Titration, and Transition from Intravenous to Oral Treprostinil in a Patient with Severe Pulmonary Arterial Hypertension. Case Reports in Pulmonology, doi: 10.1155/2015/498981.

Hambly, N. A. (2016 ). Pulmonary hypertension: diagnostic approach and optimal management. CMAJ-JAMC 188 (11), 804-812.

Hanlon, P. (2017, 9 November). Managing Prostacyclin Side Effects. Retrieved from PHA: https:// phassociation.org/managing-prostacyclin-side -effects/

McLaughlin, V. (2013). Managing pulmonary arterial hypertension and optimizing treatment options: Prognosis of pulmonary artery hypertension. . The A merican Journal of Cardiology 111 (8).

Morris KL, K. A. (2018). Outpatient Transition of Parenteral to Oral Prostacyclin in Pulmonary Arterial Hypertension. PHA Online University (p. 121). Orlando: Pulmonary Hypertension Association.

Pulmonary Hypertension Association. (2019). About Pulmonary Hypertension: The WHO Groups. Retrieved from PHA: https:/?phassociation. org/types-pulmonary-hypertension-groups/

Saito, Y. N. (2015). Epoprostenol sodium for treatment of pulmonary arterial hypertension. Vascular Health Risk Management ; 11, 265-270.

Surapaneni, P. V. (2011). Valvular Heart Disease with 
the Use of Fenfluramine-Phentermine. Texas

Heart Institute Journal, 38(5), 581-583.

United Therapeutics. (2013). United Therapeutics

Announces FDA Approval of Orenitram Ex-

tended Release Tablets for Pulmonary Arteri-

al Hypertension Treatment - Final. Fair Disclosure Wire. 